\title{
High-dose-rate brachytherapy as monotherapy for prostate cancer: technique, rationale and perspective
}

\author{
Yasuo Yoshioka, MD, PhD!, Osamu Suzuki, MD, PhD!, Yuki Otani, PhD!, Ken Yoshida, MD, PhD², Takayuki Nose, MD, PhD³, \\ Kazuhiko Ogawa, MD, PhDl \\ 'Department of Radiation Oncology, Osaka University Graduate School of Medicine, Osaka, Japan; ²Department of Radiology, Osaka \\ Medical College, Osaka, Japan, ${ }^{3}$ Department of Radiation Oncology, Nippon Medical School Tama Nagayama Hospital, Tokyo, Japan
}

\begin{abstract}
High-dose-rate (HDR) brachytherapy as monotherapy is a comparatively new brachytherapy procedure for prostate cancer. Although clinical results are not yet mature enough, it is a highly promising approach in terms of potential benefits for both radiation physics and radiobiology. In this article, we describe our technique for monotherapeutic HDR prostate brachytherapy, as well as the rationale and theoretical background, with educational intent.

Key words: prostate cancer, radiotherapy, high-dose-rate (HDR) brachytherapy, monotherapy, hypofractionation.

\section{Purpose}

Multiple treatment options are available for clinically localized prostate cancer, including radical prostatectomy, external beam radiotherapy (EBRT), brachytherapy, and a combination of EBRT and brachytherapy. Brachytherapy in the form of a permanent low-dose-rate (LDR) seed implant, or as high-dose-rate (HDR) afterloading, can deliver a highly localized radiation dose to the tumor. While LDR brachytherapy has been examined and assessed the most, and become a standard treatment option, HDR brachytherapy has recently gained momentum as an alternative. Several features of HDR brachytherapy, including uniformly accurate, precise, and reproducible dosimetry resulting from its advanced optimization capabilities, radiobiological and radioprotective advantages, as well as reduced costs, have made HDR attractive for the treatment of prostate cancer. These advantages avoid the dosimetric uncertainties of LDR related to post implant volume changes, due to needle trauma and subsequent edema during the overall treatment period of several months. High-dose-rate significantly improves the radiation dose distribution, because it can modulate and accurately control both the spatial source position and dwell time during treatment [1].

Historically, HDR brachytherapy was introduced to boost EBRT [2,3]. However, this combination typically adds 4-5 weeks to the time needed for completion of EBRT, in addition to hospitalization for HDR brachytherapy [4].
High-dose-rate brachytherapy as monotherapy, on the other hand, would definitely be the most efficient method of achieving a high degree of conformity and dose escalation. Our group was the first to report on the use of HDR brachytherapy as monotherapy [5], and subsequently reported its promising preliminary and interim outcomes [6-9]. However, to date there are only a small number of centers worldwide that carry out HDR monotherapy. Because HDR monotherapy does not include supplemental EBRT, it requires technical maturity in terms of both implant technique and treatment planning. A special patient care protocol is also needed to manage the single implant over several days of treatment. The aim of this article is to describe our technique and rationale for prostate HDR monotherapy, and to put it in perspective, with educational intent.

\section{Guidelines and recommendations on high-dose- rate brachytherapy for prostate cancer}

Clinical results for EBRT and HDR brachytherapy combination therapy have been accumulating, and they culminated in recommendations by the Groupe Européen de Curiethérapie/European Society for Radiotherapy and Oncology (GEC-ESTRO) [10,11], and consensus guidelines by the American Brachytherapy Society (ABS) [12]. The updated National Comprehensive Cancer Network (NCCN) guidelines in the USA state that HDR brachytherapy can be used in combination with EBRT (40-50 Gy) instead of 
LDR [13]. As yet, however, no guidelines or recommendations have been established on HDR monotherapy for prostate cancer, therefore it should be undertaken in clinical trial settings.

\section{Suitable patients for high-dose-rate monotherapy}

Patient selection criteria for HDR prostate monotherapy are the subject of hot debate. Although our group's initial indication for HDR monotherapy was low- to highrisk prostate cancer [5], subsequent researchers limited indications to low-risk or low-to-intermediate-risk patients [14-17]. As a result, some investigators maintained that HDR monotherapy was suitable only for low-risk or low-to-intermediate risk patients, and a combination of EBRT and HDR brachytherapy for intermediate- to high-risk patients, thus emulating the scheme for LDR brachytherapy. However, recently published reports with a relatively large number of patients revealed excellent biochemical control rates, even for intermediate- and high-risk patients [18-20], including our reports [8,9]. Moreover, considering HDR monotherapy's capability to irradiate even extracapsular lesions, we think there is no reason to limit its indications to low-risk patients, and such indications now tend to be extended to high-risk patients. Our eligibility criteria are: 1) stage T1c-T3b, or T4 with only bladder neck invasion and NOM0 as established by clinical, biochemical, and imaging studies, including magnetic resonance imaging, computed tomography (CT), and bone scans; 2) suitable candidate for epidural anesthesia; and 3) informed consent. Patients are eligible for treatment independent of gland size, provided there is a sufficiently broad pelvic inlet and freedom from lower urinary tract symptoms. Patients are considered ineligible when they have had previous pelvic radiotherapy, surgery or transurethral resection of the prostate. Characteristics of our patients (who were accrued consecutively in the clinical trial setting and for whom informed consents were obtained) are shown in Table 1, with information on accompanying hormone therapy.

\section{Details of technique}

\section{Applicator needle implant (Fig. 1A-C)}

The implant technique has been previously described in detail by our group [5]. Under epidural anesthesia, the patient is placed in a dorsal lithotomy position, with the perineal region sterilized. A balloon catheter is inserted into the bladder, with air-mixed gel placed within the prostatic urethra to enable visualization of the urethra on ultrasonography (US). Under real-time transrectal-US (TRUS) guidance, metallic applicator needles (Trocar Point Needles and Needle Stoppers ${ }^{\circledR}$; Nucletron, an Elekta company, Sweden) are placed through the perineal skin, using our in-house template. The template is made of transparent acryl with 167 needle holes spaced at $5 \mathrm{~mm}$ intervals. The needles are placed along the line that encompasses the prostate at the largest cross-section on US, except for the rectal side where the needles are placed 2-3 mm inside the prostate contour. For T3 tumors, needles can be placed outside the prostate capsule and/or into the seminal vesicles. Inner needles are inserted at $1-\mathrm{cm}$ intervals, to adequately cover the base and apex of the prostate, taking care in avoiding the urethra. The total number of needles inserted is usually around 15 . The tips of the needles are placed $2 \mathrm{~cm}$ within the bladder pouch for the reason described in the following section. We recommend placing three or four metallic fiducial markers inside the prostate gland, as far apart as possible, with at least one at the base and another at the apex. They are useful for recognizing the relative shift between the prostate and the needles, as well as deformation of the prostate itself due to edema.

\section{Treatment planning (Fig. 2A, B)}

After the implantation of the needles, CT data are acquired with the patient in the supine position (not in lithotomy). The CT slice thickness is $1.25 \mathrm{~mm}$ in helical mode. One hour before CT data acquisition and each irradiation fraction, the urinary balloon catheter is clamped in place to keep the urine within the bladder pouch, so that the cranial side of the bladder wall and the bowel are kept away from the irradiation volume.

Computed tomography-based treatment planning is performed with the aid of Oncentra ${ }^{\circledR}$ Brachy (Nucletron, an Elekta company, Sweden). The clinical target volume (CTV) includes the whole prostate gland with a 5-mm margin, except for the posterior (rectal) margin, which varies from 2 to $5 \mathrm{~mm}$ depending on the distance to the rectal wall. If extracapsular and/or seminal vesicle invasion are observed or strongly suspected, that area is included in the CTV and applicators are placed there. The planning target volume (PTV) is equal to the CTV, except in the cranial direction where it is increased by $1 \mathrm{~cm}$, and includes the bladder base. The top $2 \mathrm{~cm}$ of the applicators are placed within the bladder pouch, so that the PTV includes a $1 \mathrm{~cm}$ margin in the cranial direction around the CTV. This margin is established, not only to avoid the cold area at the base of the prostate, but also to compensate for possible needle displacement in the caudal direction.

The dose distribution is created by geometric optimization (volume method) and manual modification. The following dose constraints are applied: the dose to the whole urethra should be $100-150 \%$ of the prescription dose, preferably $<125 \%$, and the dose to the whole rectal mucosa should be $<100 \%$ of the prescription dose, preferably $<80 \%$. The PTV coverage requirements are $\mathrm{D}_{90}>100 \%$ (mandatory) and $\mathrm{V}_{100}>97 \%$ (preferable). The dose-volume constraint for the rectum is $D_{5 c c}<55 \%$, which is drawn from our previous analysis, where $\mathrm{D}_{5 \mathrm{cc}}<27$ Gy is a significant cut-off value for late rectal toxicity [21]. The biologically effective dose (BED) of 27 Gy in 9 fractions in the previous analysis corresponded to $55 \%$ of the prescription dose in our ongoing dose-fractionation of 45.5 Gy in 7 fractions, assuming an $\alpha / \beta$ value for rectal mucosa of $3 \mathrm{~Gy}$.

\section{Patient management}

The patient remains in bed for 4 days from Monday (implant day: Day 1) to Thursday (removal day: Day 4) 

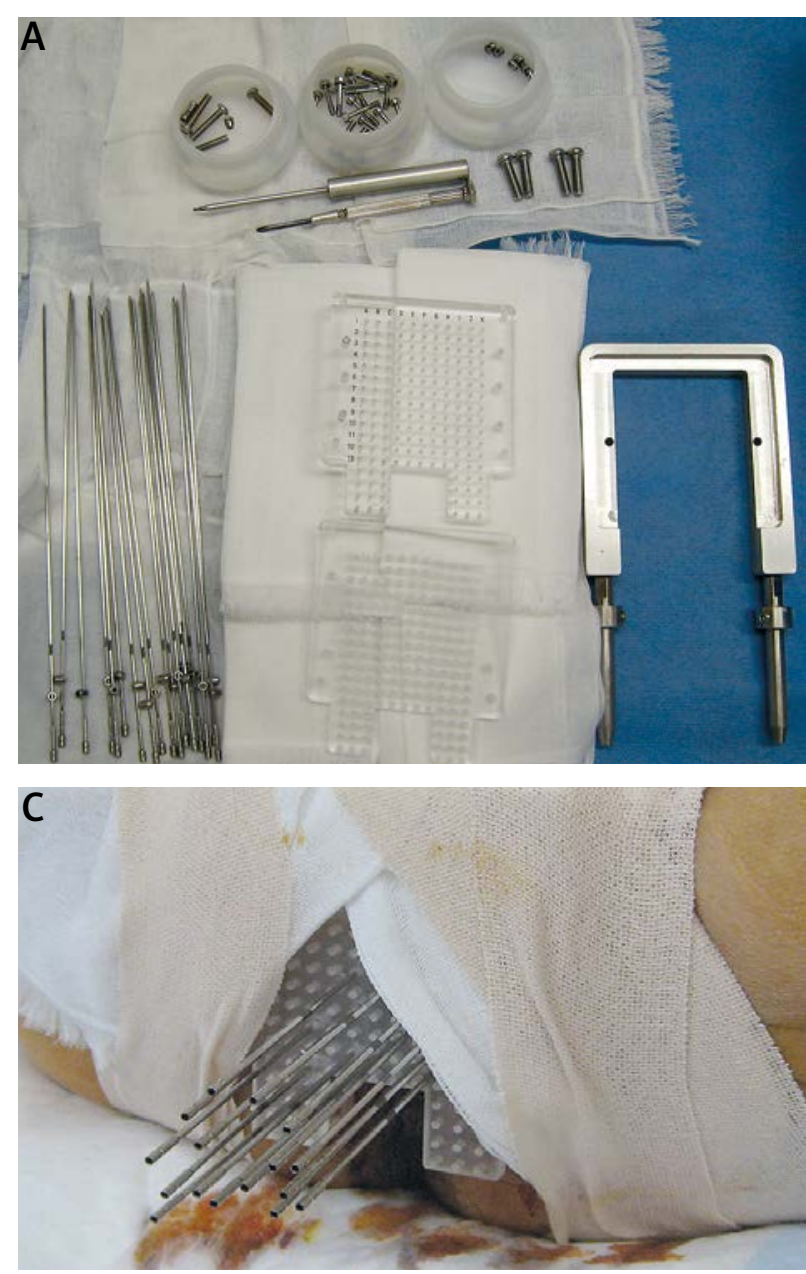

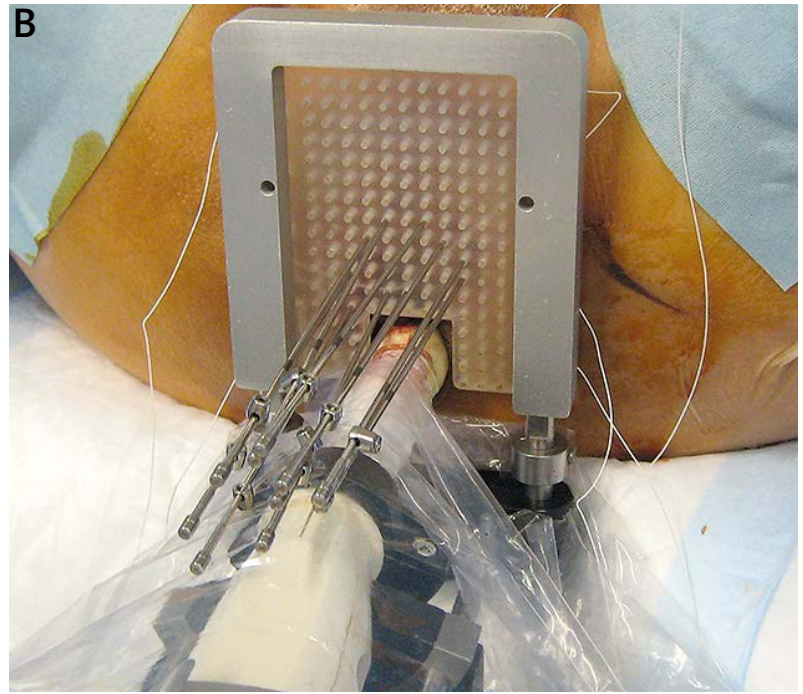

Fig. 1. A) Preparation for implant of applicator needles for HDR prostate brachytherapy. An in-house "see-through" template and its cover plate (center), an in-house metallic frame to hold and connect the template with an ultrasonography-probe stepper (right), applicator needles with stoppers (left), and their screws and screw drivers (upper). B) Needle implant under real-time transrectal-ultrasonography guidance. The patient is awake, under epidural anesthesia, in lithotomy position. Template holes had been superimposed on the ultrasonography monitor. C) Fixation of the template with elastic tape. Before taping, the template had been sutured to the perineal skin. Needle stoppers are sandwiched by the template and its cover plate, preventing needle displacement
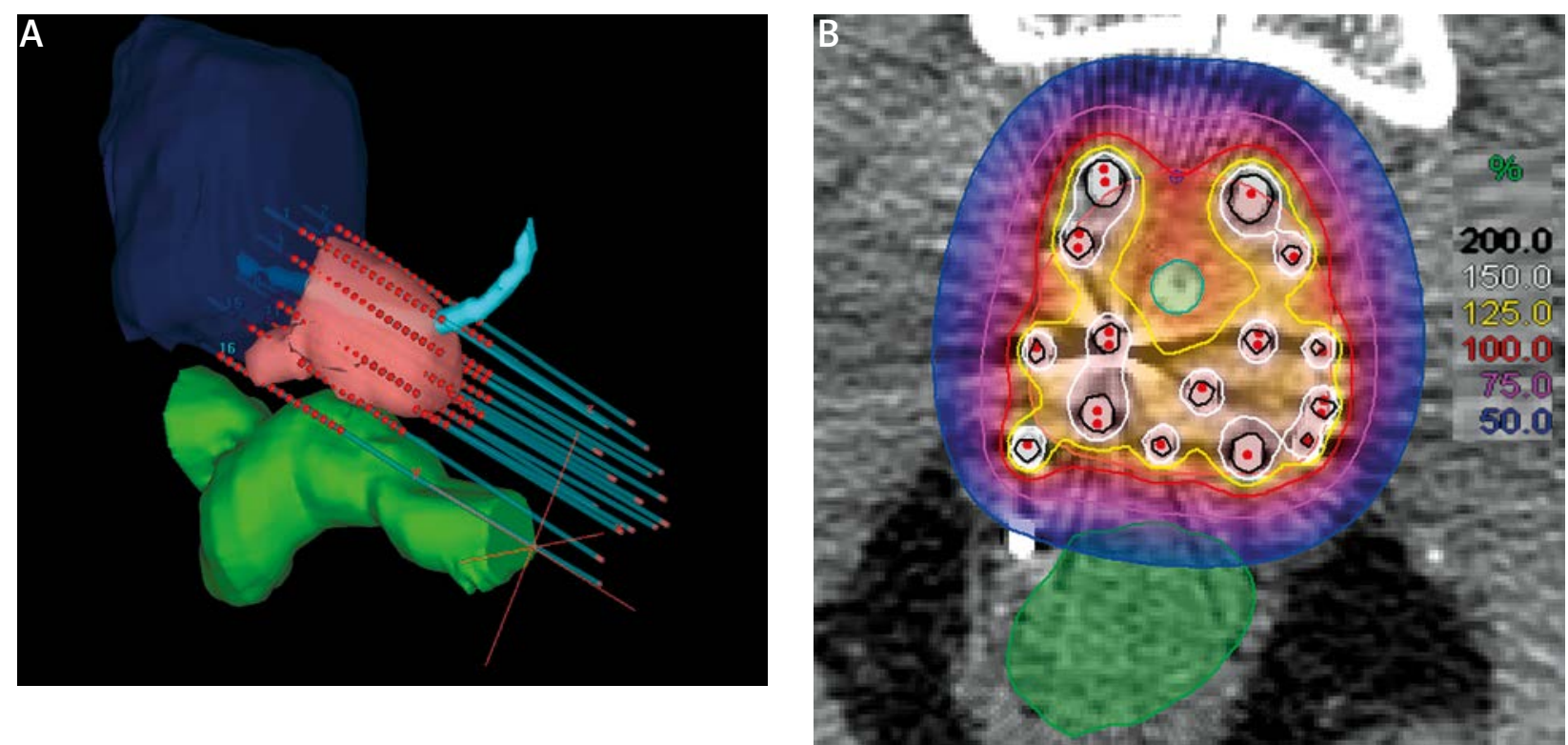

Fig. 2. A) Three-dimensional reconstruction of the prostate and proximal seminal vesicles (pink), rectum (green), bladder (blue), urethra (cyan), and applicator needles and source dwell positions (red). Dwell positions were automatically selected by designating the area up to 7-mm outside the prostate or seminal vesicles. Note that some needles and dwell positions were entirely outside the prostate grand and/or partly in the seminal vesicles or in the bladder pouch. B) A dose distribution plot of transverse plane. Note that the urethral dose was $<125 \%$ of the prescription dose, and the rectal dose $<75 \%$. Most parts of the rectum received $<50 \%$ of the prescription dose, which would be difficult to achieve with EBRT (even with IMRT) 
Table 1. Patient characteristics at Osaka University Hospital

\begin{tabular}{|c|c|c|c|}
\hline Years & 1995-1996 & $1996-2005$ & $2005-2010^{*}$ \\
\hline Dose-fractionation & $48 \mathrm{~Gy} / 8 \mathrm{fr} / 5$ days & 54 Gy/9 fr/5 days & $45.5 \mathrm{~Gy} / 7 \mathrm{fr} / 4$ days \\
\hline Number of patients & 7 & 112 & 63 \\
\hline Age median (range) & $67(45-78)$ & $68(47-81)$ & $69(50-82)$ \\
\hline \multicolumn{4}{|l|}{ T classification: } \\
\hline $\mathrm{T} 1$ & 0 & 28 & 15 \\
\hline $\mathrm{T} 2$ & 1 & 34 & 32 \\
\hline T3 & 4 & 46 & 14 \\
\hline T4 & 2 & 4 & 2 \\
\hline \multicolumn{4}{|l|}{ Gleason score: } \\
\hline$\leq 6$ & 1 & 50 & 11 \\
\hline 7 & 1 & 36 & 34 \\
\hline$\geq 8$ & 0 & 26 & 18 \\
\hline \multicolumn{4}{|l|}{ Pretreatment PSA $(\mathrm{ng} / \mathrm{ml})$ : } \\
\hline$<10.0$ & 1 & 31 & 26 \\
\hline $10.0-20.0$ & 0 & 31 & 22 \\
\hline$\geq 20.0$ & 6 & 50 & 15 \\
\hline Median (range) & $36.3(7.0-150.0)$ & $16.6(3.8-233.0)$ & $11.5(3.9-378.5)$ \\
\hline \multicolumn{4}{|l|}{ Risk group**: } \\
\hline Low & 0 & 15 & $--^{\star \star \star}$ \\
\hline Intermediate & 1 & 29 & 34 \\
\hline High & 6 & 68 & 29 \\
\hline \multicolumn{4}{|l|}{ Hormone therapy } \\
\hline Low & - & $9(60 \%)$ & - \\
\hline Median duration (range) (mo) & - & $16(5-36)$ & - \\
\hline Intermediate & $0(0 \%)$ & $19(66 \%)$ & $12(35 \%)$ \\
\hline Median duration (range) (mo) & - & $12(3-156)$ & $7(1-24)$ \\
\hline High & $5(83 \%)$ & $66(97 \%)$ & $25(86 \%)$ \\
\hline Median duration (range) (mo) & $54(45-180)$ & $43(2-188)$ & $24(4-94)$ \\
\hline \multicolumn{4}{|l|}{ Follow-up (years) } \\
\hline Median (range) & $6.8(3.3-17.4)$ & $5.4(1.3-11.4)$ & $3.5(1.1-6.0)$ \\
\hline
\end{tabular}

under continuous epidural anesthesia, and undergoes irradiation twice daily with an interval of $\geq 6$ hours. The treatment consists of 7 fractions of 6.5 Gy each (total $45.5 \mathrm{~Gy}$ ). Anticoagulated patients are told to stop their drugs 1-2 weeks before the implant. To suppress defecation, the patients are given low-residue meals from Day 1 to Day 4 . The patients should be given purgatives starting 4 days before the implant (magnesium oxide for 3 days and sennoside the night before) and a glycerin enema early in the morning of Day 1 . The patients should also be encouraged to defecate before the implant. Prophylactic antibiotics are administered intravenously on Day 1 and Day 4, at the time of needle insertion and removal, and orally 3 times daily on Day 2 and Day 3. Pneumatic compression devices are attached to the patients' lower legs to prevent deep vein thrombosis from Day 1 through Day 4. To minimize bleeding (both from the perineum and intravesically), a coagulating agent (carbazochrome) is ad- ministered through an intravenous drip at the time of the implant and at the time of needle removal. Immediately after pulling the needles out, the physician should manually compress the prostate using both hands, one via the perineum and the other via the rectum (as in a digital examination), to stop the bleeding. In addition, pulling the balloon catheter, which has been replaced with a larger 3-way catheter for bladder irrigation, with the balloon inflated to its maximum, helps to stop bleeding from the bladder neck. For intravesical bleeding, bladder irrigation with cold saline is effective, and the continuous bladder irrigation technique is used to prevent clots from occluding the balloon catheter when intravesical bleeding is protracted.

\section{Neoadjuvant and/or adjuvant hormone therapy}

The benefits of neoadjuvant and/or adjuvant hormone therapy are controversial. We assume that the therapy's 


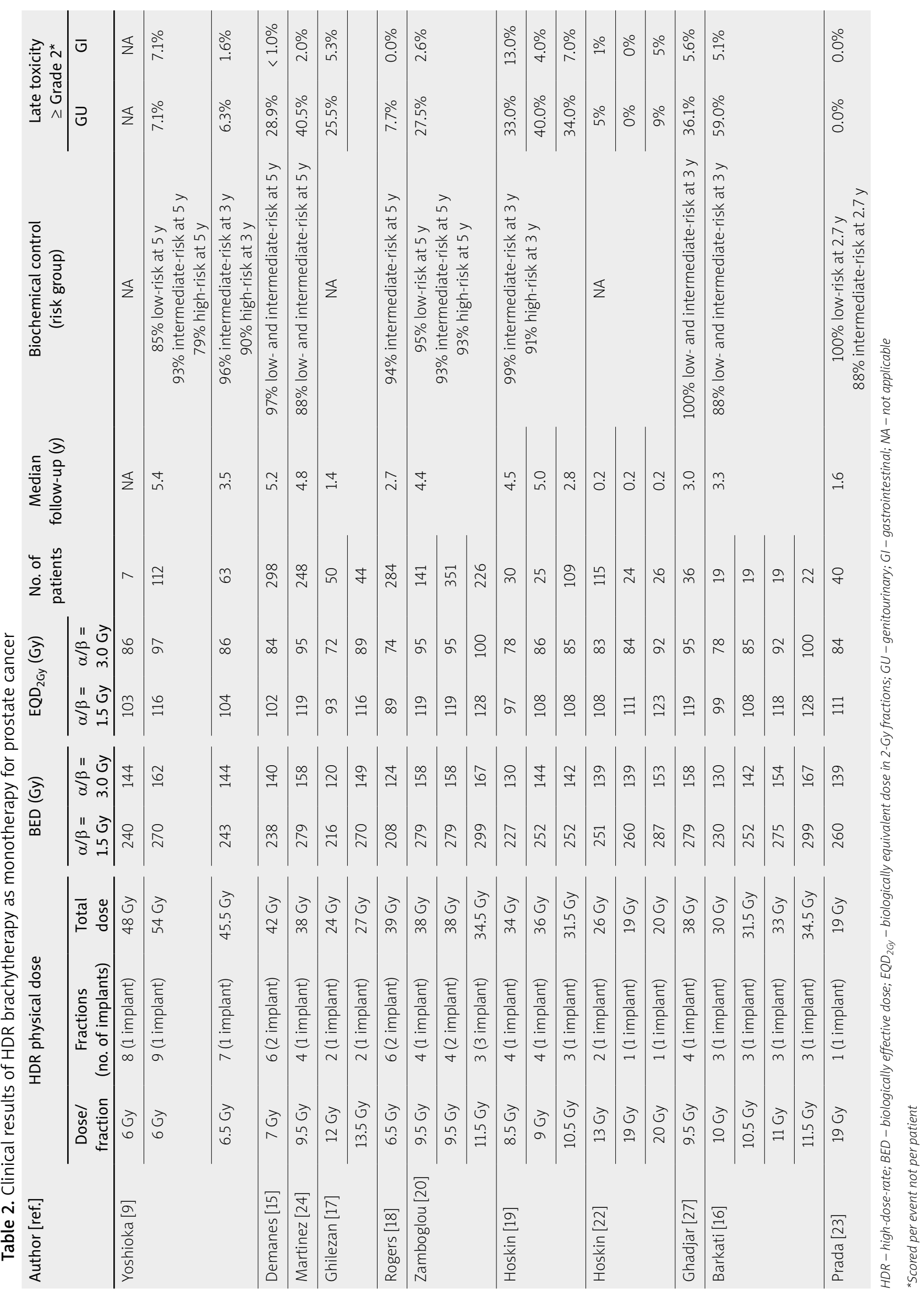


additional benefit over RT alone would be smaller in the case of HDR brachytherapy than for EBRT (for example, classical 70-Gy EBRT), because BED of HDR brachytherapy is far higher than that of EBRT. However, some interaction between hormones and radiation may still occur, and the volume reduction effect may be associated with less toxicity. In our present protocol, patients with only one intermediate-risk feature are not given hormone therapy. The other intermediate-risk and all high-risk patients receive 6 to 12 months of neoadjuvant hormone therapy, but no adjuvant. If high-risk patients prefer to have long-term hormone therapy after being informed of its survival benefit in the case of EBRT, then adjuvant hormone therapy is allowed for up to 3 years total duration.

\section{Patient outcomes}

Table 2 lists clinical results of monotherapeutic HDR brachytherapy for prostate from the literature [5-7,15-20, 22-27]. Only 10 institutions worldwide have reported clinical results for prostate HDR monotherapy. The longest median follow-up was 5.4 years, which was reported from our institution [8], while the median follow-up of most of the studies was only 1-3 years.

The reported 5-year prostate-specific antigen (PSA) control rate for low-risk groups ranged from $85-97 \%$, mostly $>90 \%$. For intermediate-risk groups, some authors reported a PSA control rate of 93-94\%, and for highrisk groups, it was reported between 79 and $93 \%$, mostly $>80 \%$. Although none of these studies have reported a follow-up period much beyond 5 years, the overall PSA control rates reported thus far have been excellent, which may be attributed to the high BED of $>200$ Gy discussed

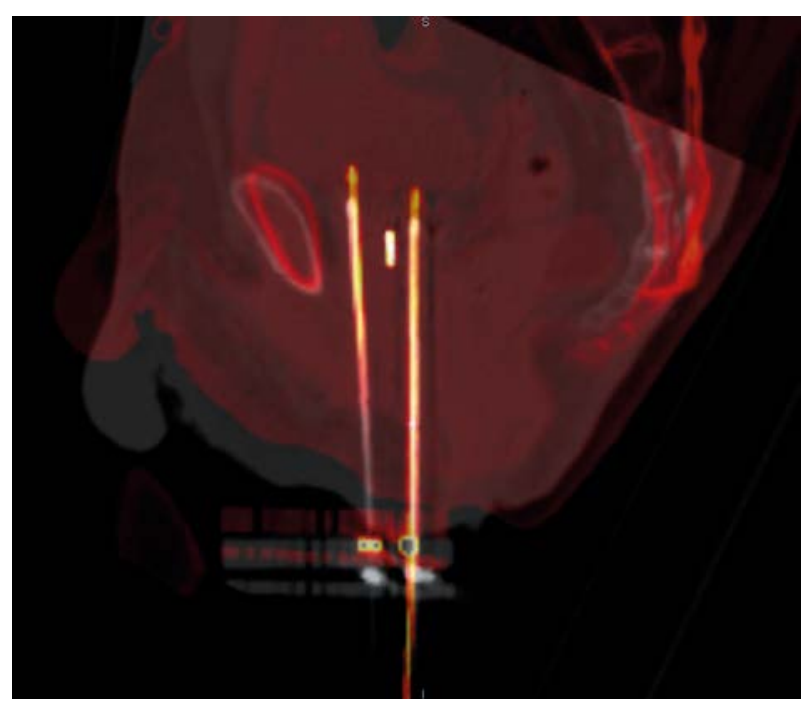

Fig. 3. Treatment planning CT on the implant day (magenta) and on the last irradiation day (Day 4, gray), which were overlaid by matching positions of the metallic fiducial marker (VISICOIL ${ }^{\circledR}$ ). Note that the geometry of needle fiducial template was kept constant, in contrary to the shift of pubic symphysis or sacral bone. However, the needles moved about $1 \mathrm{~cm}$ in the caudal direction, together with the template, which might be attributable to the perineal edema in the following section. The reported toxicity levels were generally acceptable (Table 2).

\section{Advantages/disadvantages of high-dose-rate monotherapy}

Advantages and disadvantages of prostate HDR monotherapy, compared to other treatment options, have been detailed in our review article published elsewhere [28]. Below are the summaries, including (1-4) description of advantages with respect to radiation physics, (5) advantages with respect to radiobiology, and (6) disadvantages: 1. Medical staff are never exposed to radiation and patients can stay in a regular ward since there is no need for a shielded room. Patients only need to go to an HDR unit room for irradiation for 30 minutes per fraction.

2. Treatment planning is based on the CT images obtained after needle insertion, or on the TRUS images obtained at the time of needle insertion. The dwell positions of the stepping source are determined in terms of real anatomy. The dwell time for each dwell position is then calculated with an optimization algorithm.

3. Unlike for EBRT, inter-/intra-fraction organ motion is not a problem with HDR brachytherapy. In the case of EBRT, several factors including daily set-up errors, retention of feces, gas, or urine, respiratory motion, or peristaltic motion result in discrepancies between the coordinates of the tumor and the radiation beam. With brachytherapy, these two coordinates are always concordant, because the tumor and the radioactive sources move in unison, so that PTV is normally identical to CTV. The overall treatment time for HDR monotherapy typically ranges from 1-4 days, significantly shorter than for EBRT.

4. Unlike for LDR brachytherapy, HDR brachytherapy needles can be placed at the extracapsular lesion, and even into the seminal vesicles and/or into the bladder pouch. The cable-connected stepping source simply moves back and forth within the closed space without any risk of source migration or dropping out. Therefore, the indication for HDR monotherapy can potentially even be extended to $\mathrm{T} 3 \mathrm{a} / \mathrm{b}$ or some $\mathrm{T} 4$ tumors. The dwell time optimization makes a significant urethral dose reduction possible for HDR compared to that for LDR.

5. Recent findings that the $\alpha / \beta$ value for prostate cancer is less than that for the surrounding late-responding normal tissue have made hypofractionation attractive, and HDR monotherapy can maximize this advantage of hypofractionation [29-33]. Table 2 lists dose-fractionations and associated BED and EQD 2 Gy of HDR monotherapy from the literature. The BED for prostate cancer ranges from 208-299 Gy, with a median of $256 \mathrm{~Gy}$. The values for $\mathrm{EQD}_{2 \mathrm{~Gy}}$ range from $89-128$ Gy with a median of $110 \mathrm{~Gy}$, which may be impossible to administer with EBRT, even with IMRT. As for late toxicity, $\mathrm{EQD}_{2 \mathrm{~Gy}}$ ranges from 72-110 Gy, with a median of $86 \mathrm{~Gy}$, which can be considered the equivalent of the maximum dose of 86.4 Gy administered with the current IMRT. This means that, theoretically, hypofrac- 
tionation with a large fraction size can enhance BED for prostate cancer without increasing BED for late-responding tissue.

6. One of the possible drawbacks of HDR brachytherapy is the problem of applicator needle displacement during treatment (Fig. 3), which has been pointed out by some groups [14,34-41]. However, this problem does not arise if there is one fraction per implant [20-23]. Another drawback of HDR is the requirement of hospitalization and patients having to stay in bed during the treatment period. Further potential disadvantages of HDR are tumor edema and bleeding from needle insertion, causing changes in dosimetry from planning to treatment. In addition, if large doses are given to normal tissues (perhaps in less experienced centers), there is an increased risk of late toxicity, given the large dose per fraction.

\section{Future directions}

High-dose-rate prostate monotherapy is still evolving and being developed, with ongoing research to determine optimal methodology and dose-fractionations. At our affiliated hospital, a new technique enabling more accurate implantation into seminal vesicles, and a new ambulatory technique for multi-fraction HDR brachytherapy have been developed $[42,43]$. In view of its high degree of freedom, there may still be some room for improvement in the dwell time optimization algorithm [44,45]. As for the problem of needle displacement, we are now testing daily CT scans to adjust needle positions or source dwell positions, by means of readjusting the relative locations of dwell positions to the gravity of the implanted fiducial markers. However, this method causes additional radiation exposure to the patient, which should be taken in to account as an intrinsic disadvantage.

Recent trends are moving towards a smaller number of fractions and shorter treatment. Many institutions previously used 4 fractions or more $[5,14,15,18]$. However, researchers recently reported 3 fractions with 3 implants (1 fraction per implant) [20], 2 fractions in a single day [17], and even single fraction HDR monotherapy [22,23]. Such an extremely hypofractionated regimen would maximize the therapeutic ratio, and at the same time avoid the HDR brachytherapy drawbacks of hospitalization and needle displacement during the treatment period. Finally, the authors would like to emphasize that HDR monotherapy has the largest potential to control prostate cancer without compromising toxicity, from the perspective of both radiation physics and radiobiology. Further clinical research is therefore clearly warranted.

\section{Disclosure}

Authors report no conflict of interest.

\section{References}

1. Skowronek J. Low-dose-rate or high-dose-rate brachytherapy in treatment of prostate cancer - between options. J Contemp Brachytherapy 2013; 5: 33-41.

2. Galalae RM, Kovács G, Schultze J et al. Long-term outcome after elective irradiation of the pelvic lymphatics and local dose escalation using high-dose-rate brachytherapy for locally advanced prostate cancer. Int J Radiat Oncol Biol Phys 2002; 52: 81-90.

3. Mate TP, Gottesman JE, Hatton J et al. High dose-rate afterloading Ir-192 prostate brachytherapy: feasibility report. Int J Radiat Oncol Biol Phys 1998; 41: 525-533.

4. Hijazi H, Chevallier D, Gal J et al. Prostate cancer boost using high-dose rate brachytherapy: early toxicity analysis of 3 different fractionation schemes. J Contemp Brachytherapy 2013; 5: 203-209.

5. Yoshioka Y, Nose T, Yoshida K et al. High-dose-rate interstitial brachytherapy as a monotherapy for localized prostate cancer: treatment description and preliminary results of a phase I/II clinical trial. Int J Radiat Oncol Biol Phys 2000; 48: 675-681.

6. Yoshioka Y, Nose T, Yoshida K et al. High-dose-rate brachytherapy as monotherapy for localized prostate cancer: a retrospective analysis with special focus on tolerance and chronic toxicity. Int J Radiat Oncol Biol Phys 2003; 56: 213-220.

7. Yoshioka Y, Konishi K, Oh RJ et al. High-dose-rate brachytherapy without external beam irradiation for locally advanced prostate cancer. Radiother Oncol 2006; 80: 62-68.

8. Yoshioka Y, Konishi K, Sumida I et al. Monotherapeutic high-dose-rate brachytherapy for prostate cancer: Five-year results of an extreme hypofractionation regimen with $54 \mathrm{~Gy}$ in nine fractions. Int J Radiat Oncol Biol Phys 2011; 80: 469-475.

9. Yoshioka Y, Konishi K, Suzuki O et al. Monotherapeutic high-dose-rate brachytherapy for prostate cancer: a dose reduction trial. Radiother Oncol 2014; 110: 114-119.

10. Kovács G, Pötter R, Loch T et al. GEC/ESTRO-EAU recommendations on temporary brachytherapy using stepping sources for localised prostate cancer. Radiother Oncol 2005; 74: 137-148.

11. Hoskin PJ, Colombo A, Henry A et al. GEC/ESTRO recommendations on high dose rate afterloading brachytherapy for localised prostate cancer: an update. Radiother Oncol 2013; 107: 325-332.

12. Yamada Y, Rogers L, Demanes DJ et al; American Brachytherapy Society. American Brachytherapy Society consensus guidelines for high-dose-rate prostate brachytherapy. Brachytherapy 2012; 11: 20-32.

13. National Comprehensive Cancer Network (NCCN) Clinical Practice Guidelines in Oncology. Prostate Cancer. Version 4. 2013. http://www.nccn.org/professionals/physician_gls/pdf/ prostate.pdf; accessed: Nov. 20, 2013.

14. Martinez A, Pataki I, Edmundson G et al. Phase II prospective study of the use of conformal high-dose-rate brachytherapy as monotherapy for the treatment of favorable stage prostate cancer: a feasibility report. Int J Radiat Oncol Biol Phys 2001; 49: 61-69.

15. Demanes DJ, Martinez AA, Ghilezan M et al. High-dose-rate monotherapy: safe and effective brachytherapy for patients with localized prostate cancer. Int J Radiat Oncol Biol Phys 2011; 81: 1286-1292.

16. Barkati M, Williams SG, Foroudi F et al. High-dose-rate brachytherapy as a monotherapy for favorable-risk prostate cancer: a phase II trial. Int J Radiat Oncol Biol Phys 2012; 82: 1889-1896.

17. Ghilezan M, Martinez A, Gustason G et al. High-dose-rate brachytherapy as monotherapy delivered in two fractions within one day for favorable/intermediate-risk prostate cancer: preliminary toxicity data. Int J Radiat Oncol Biol Phys 2012; 83: 927-932.

18. Rogers CL, Alder SC, Rogers RL et al. High dose brachytherapy as monotherapy for intermediate risk prostate cancer. J Urol 2012; 187: 109-116. 
19. Hoskin P, Rojas A, Lowe G et al. High-dose-rate brachytherapy alone for localized prostate cancer in patients at moderate or high risk of biochemical recurrence. Int J Radiat Oncol Biol Phys 2012; 82: 1376-1384.

20. Zamboglou N, Tselis N, Baltas D et al. High-dose-rate interstitial brachytherapy as monotherapy for clinically localized prostate cancer: treatment evolution and mature results. Int J Radiat Oncol Biol Phys 2013; 85: 672-678.

21. Konishi K, Yoshioka Y, Isohashi F et al. Correlation between dosimetric parameters and late rectal and urinary toxicities in patients treated with high-dose-rate brachytherapy used as monotherapy for prostate cancer. Int J Radiat Oncol Biol Phys 2009; 75: 1003-1007.

22. Hoskin P, Rojas A, Ostler P et al. High-dose-rate brachytherapy alone given as two or one fraction to patients for locally advanced prostate cancer: acute toxicity. Radiother Oncol 2014; 110: 268-271.

23. Prada PJ, Jimenez I, González-Suárez H et al. High-dose-rate interstitial brachytherapy as monotherapy in one fraction and transperineal hyaluronic acid injection into the perirectal fat for the treatment of favorable stage prostate cancer: treatment description and preliminary results. Brachytherapy 2012; 11: 105-110.

24. Martinez AA, Demanes J, Vargas C et al. High dose rate prostate brachytherapy: an excellent accelerated hypofractionated treatment for favorable prostate cancer. Am J Clin Oncol 2010; 33: 481-488.

25. Martin T, Baltas D, Kureck R et al. 3D conformal HDR brachytherapy as monotherapy for localized prostate cancer. A pilot study. Strahlenther Oncol 2004; 180: 225-232.

26. Corner C, Rajas AM, Bryant L et al. A Phase II study of high dose rate afterloading brachytherapy as monotherapy for the treatment of localized prostate cancer. Int J Radiat Oncol Biol Phys 2008; 72: 441-446.

27. Ghadjar P, Keller T, Pentsch CA et al. Toxicity and early treatment outcomes in low and intermediate-risk prostate cancer managed by high-dose rate brachytherapy as monotherapy. Brachytherapy 2009; 8: 45-51.

28. Yoshioka Y, Yoshida K, Yamazaki H et al. The emerging role of high-dose-rate (HDR) brachytherapy as monotherapy for prostate cancer. J Radiat Res 2013; 54: 781-788.

29. Brenner DJ, Hall EJ. Fractionation and protraction for radiotherapy of prostate carcinoma. Int J Radiat Oncol Biol Phys 1999; 43: 1095-1101.

30. Fowler J, Chappell R, Ritter M. Is alpha/beta for prostate tumors really low? Int J Radiat Oncol Biol Phys 2001; 50: 1021-1031.

31. Brenner DJ, Martinez AA, Edmundson GK et al. Direct evidence that prostate tumors show high sensitivity to fractionation (low alpha/beta ratio), similar to late-responding normal tissue. Int J Radiat Oncol Biol Phys 2002; 52: 6-13.

32. Wang JZ, Guerrero M, Li XA. How low is the alpha/beta ratio for prostate cancer? Int J Radiat Oncol Biol Phys 2003; 55 194-203.

33. Miralbell R, Roberts SA, Zubizarreta E et al. Dose-fractionation sensitivity of prostate cancer deduced from radiotherapy outcomes of 5,969 patients in seven international institutional datasets: $\alpha / \beta=1.4(0.9-2.2)$ Gy. Int J Radiat Oncol Biol Phys 2012; 82: e17-24.

34. Damore SJ, Syed AM, Puthawala AA et al. Needle displacement during HDR brachytherapy in the treatment of prostate cancer. Int I Radiat Oncol Biol Phys 2000; 46: 1205-1211.

35. Hoskin PJ, Bownes PJ, Ostler P et al. High dose rate afterloading brachytherapy for prostate cancer: Catheter and gland movement between fractions. Radiother Oncol 2003; 68: 285-288.

36. Mullokandov E, Gejerman G. Analysis of serial CT scans to assess template and catheter movement in prostate HDR brachytherapy. Int J Radiat Oncol Biol Phys 2004; 58: 1063-1071.
37. Simnor T, Li S, Lowe G et al. Justification for inter-fraction correction of catheter movement in fractionated high doserate brachytherapy treatment of prostate cancer. Radiother Oncol 2009; 93: 253-258.

38. Yoshida K, Yamazaki H, Nose T et al. Needle applicator displacement during high-dose-rate interstitial brachytherapy for prostate cancer. Brachytherapy 2010; 9: 36-41.

39. Foster W, Cunha JA, Hsu IC et al. Dosimetric impact of interfraction catheter movement in high-dose rate prostate brachytherapy. Int J Radiat Oncol Biol Phys 2011; 80: 85-90.

40. Kolkman-Deurloo IK, Roos MA, Aluwini S. HDR monotherapy for prostate cancer: A simulation study to determine the effect of catheter displacement on target coverage and normal tissue irradiation. Radiother Oncol 2011; 98: 192-197.

41. Takenaka T, Yoshida K, Ueda M et al. Assessment of daily needle applicator displacement during high-dose-rate interstitial brachytherapy for prostate cancer using daily CT examinations. J Radiat Res 2012; 53: 469-474.

42. Yoshida K, Nose T, Shiomi H et al. New ambulatory implant technique of high-dose-rate interstitial brachytherapy for prostate cancer. Radiat Med 2006; 24: 595-599.

43. Yoshida K, Kuroda S, Yoshida M et al. New implant technique for separation of the seminal vesicle and rectal mucosa for high-dose-rate prostate brachytherapy. Brachytherapy 2007; 6: 180-186.

44. Yoshioka Y, Nishimura T, Kamata M et al. Evaluation of anatomy-based dwell position and inverse optimization in high-dose-rate brachytherapy of prostate cancer: a dosimetric comparison to a conventional cylindrical dwell position, geometric optimization, and dose-point optimization. Radiother Oncol 2005; 75: 311-317.

45. Sumida I, Shiomi H, Yoshioka Y et al. Optimization of dose distribution for HDR brachytherapy of the prostate using Attraction-Repulsion Model. Int J Radiat Oncol Biol Phys 2006; 64: 643-649. 